Journal of Universal Computer Science, vol. 26, no. 8 (2020), 1017-1042

submitted: 30/1/2020, accepted: 13/7/2020, appeared: 28/8/2020 CC BY-ND 4.0

\title{
A Systematic Review of Research about Game-based Learning in Virtual Worlds
}

\author{
Nikolaos Pellas \\ (University of Western Macedonia, Kastoria, Greece \\ aff00192@uowm.gr) \\ Stylianos Mystakidis \\ (University of Patras, Patra, Greece \\ smyst@upatras.gr)
}

\begin{abstract}
While a substantial body of literature has well-documented and demonstrated considerable potentials of virtual worlds (VWs) across a variety of learning subjects, little is known today regarding game-based learning (GBL) approaches that can be applied. This systematic literature review presents the current state of knowledge and practice about GBL approaches in VWs from Primary and Secondary (K-12) to Higher education (HE). It provides guidance for instructional designers and scholars by systematically appraising and summarizing the most relevant existing research articles published from 2006 until December 2019. Twentyeight studies were finally included for a detailed analysis and synthesis during the selection and screening process. The results indicated that GBL in VWs gained popularity from 2014 until 2016. Many studies in K-12 and HE settings were focused on describing and evaluating the appropriateness or the effectiveness of the applied instructional design processes using various game prototypes to disseminate their findings on user experience, usability issues, students' outcomes, and/or learning performance. This study contributes by reviewing how GBL approaches in VWs can potentially benefit students' learning performance, leading to a higher level of satisfaction and dimensions of disciplinary understanding. It also proposes six concrete recommendations guiding game design and development to support learning in VWs.
\end{abstract}

Keywords: game-based learning, game design, k-12 education, higher education, virtual worlds Categories: H.5.2., K.3.1, K.3.2, L.3.1, L.3.6, L.5.1

\section{Introduction}

The breadth of game-based learning (GBL) has gained significant ground due to the development and utilization of contemporary interactive environments from Primary and Secondary (K-12) to Higher education (HE). Accordingly, GBL can provide several reasonable potentials that have given to instructors and educators the chance to apply innovative forms of teaching and support the learning experience through (realistic) simulated problem-based tasks in favor of fostering students' cognitive thinking skills, such as problem-solving, critical thinking, logical reasoning, and creativity [Prensky 2007].

To have the appropriate learning conditions inside a computer game, software designers and developers need to provide playable conditions from a wide range of tasks with a deep level of interactivity. Players should be engaged in certain tasks of 
increasing difficulty to accomplish specific objectives and test the appropriateness of outcomes generated by their decisions and actions inside it. Significant works [Gee 2007; Prensky 2007] in the field of GBL stated the importance of the gameplay's contextualization when it is explicitly designed to support learning in playable and enjoyable settings. The design and development of learning tasks in game-based contexts requires a multi-dimensional approach, in which students are players who need: a) to observe specific conceptual embodiment in visual contexts that can foster their problem-solving abilities in various discovery processes guided by the instructor(s), and b) to develop cognitive thinking and practical skills to improve their learning outcomes and experience by interacting with in-game digital elements or objects.

The abundance of resources in Web 2.0 alongside the realistic simulated representational fidelity of visual objects/elements generated by three-dimensional (3D) computer graphics has created unprecedented opportunities for teaching and learning. A virtual world (VW) is a computer-supported and simulated 3D environment that is accessible by multiple users. Each user has embodied presence as an avatar (i.e. a digital representation with human or non-human characteristics) to interact in realtime and explore its features using visual objects, participate in a wide range of simulated activities or tasks, and communicate with other (or not) peers at the same time and place in a 3D online environment that is displayed on a computer monitor [Girvan, 2018]. Three major categories of VWs are as follows: (a) "metagames," such as Active Worlds, Quest Atlantis [Barab et al. 2005], (b) "social," such as Minecraft or Second Life [Mystakidis \& Berki 2018], and (c) “open-source," such as OpenSimulator or Open Wonderland [Pellas 2014].

An alternative and worthwhile approach that gained prominence in recent years is the development and creation of computer games in VWs. VWs provide a large number of tools which are easy-to-use for designing and developing game prototypes. Users can communicate with other peers asynchronously with specific tools, like gestures or IM, and synchronously, like VoIP calls to (co-)design and/or program visual objects with geometric shapes using the scripting language of VWs or the visual palette of Scratch4SL [Pellas 2014]. Game-based learning has the potential to fulfil students' learning needs and experiences by supporting various learning tasks that correspond to an imitation of an operation or of a process consisted of specific simulated real-world situations [Mystakidis et al. 2019]. Thus, a game prototype made in VW comprises specific rules, mechanisms, and objectives that can permit users as avatars to participate actively having specific task information and "learn by doing" within interactive and playable problem-solving contexts [Pellas \& Vosinakis 2018].

To date, several studies utilized GBL in VWs. A total of nineteen studies have utilized VWs in K-12 subjects including of History [Wang et al. 2018], Life Science topics [Barab et al. 2005; Dede et al. 2017; Ketelhut 2007; Lim et al. 2006; Loula et al. 2014; Metcalf et al. 2018; Tüzün, 2007], Computer science [Jakos \& Verber, 2016; Pellas 2014; Pellas \& Peroutseas 2016; Pellas \& Vosinakis 2018; Rico et al. 2011], Information Technology [Barab et al. 2012; Twining 2009; Young et al. 2012], Language learning [Zheng et al. 2009], and Mathematics [Kim \& Ke 2016; Şimşek 2016]. Additionally, a total of nine studies applied in several learning tasks using various VWs to develop game prototypes in different HE subjects encompassing Computer science [Christopoulos et al. 2018; Granic et al. 2019], Engineering [August et al. 2016; Callaghan et al. 2013], Language learning [Berns et al. 2013], Economics 
[Hornik \& Thornburg 2010], Medical education [Toro-Troconis \& Mellström 2010], and Instructional design [Franetovic 2016; Yilmaz \& Cagiltay 2016].

A growing body of literature has come to the conclusion that VWs can increase students' learning motivation and improve the quality of learning experience for various reasons. Dalgarno and Lee [2010] suggested a number of affordances that VWs can offer in contrast to other 2D platforms. These are enhanced spatial knowledge representation to assist the development of several tasks, experiential learning to practice in visually-rich hands-on tasks, increased motivation/engagement, improved contextualization of abstract concepts and a 3D environment, in which users can communicate and construct something meaningful in collaborative (or not) instructional design settings. Additionally, several researchers have utilized such affordances in alignment with theoretical underpinnings to enhance students' cognitive development provided by Constructionism [Pellas 2014], Situated learning theory [Ketelhut et al. 2007], and Embodied cognition [Zheng et al. 2009]. Summarizing the findings from the reported research, the major benefits focused on the use of GBL approaches come into the following forms: a) enhance spatial knowledge representation in problem-solving contexts [Twining, 2009], b) promote students' learning motivation and interest [Young et al. 2012], c) increase students' positive attitudes and engagement with learning content that is enhanced perceptually and semantically into $3 \mathrm{D}$ visuallyrich spaces [Barab et al. 2012], d) improve students' learning performance and outcomes to transfer learning materials into simulated real-life applications [Kim \& Ke 2016], e) allow visualizations and hands-on experiment in cross-disciplinary STEM settings [August et al. 2016], and f) encourage exploratory tasks, in which students need to use cognitive skills related to higher-order, critical thinking, and computational thinking [Pellas \& Vosinakis 2018].

Over the last ten years, several studies were published to review the relevant literature of VWs in education. However, none of these focused on the use of GBL in VWs for K-12 and HE settings from previous works published from 2008 until 2019. For example, Hew and Cheung [2010] paid attention to the systematic analysis of studies published from 2008 to 2010 and related to university and polytechnic settings without referring what specific modifications were made for instructional support. Wang and Burton [2012] analysed the descriptive findings of 107 studies, which exclusively utilised Second Life as learning platform and conducted in college settings. Pellas et al. [2017] focused on the description of instructional design frameworks and settings adopted in VWs for different STEM learning subjects. Lastly, Ghanbarzadeh and Ghapanchi [2018] investigated VWs applications in HE settings from 165 papers and reported findings from different disciplines over the last decade.

Although the above-mentioned reviews provided important aspects and considerations about the use of VWs in education, there was no explicit focus identified regarding the description of using GBL approaches in a systematic manner. Furthermore, there are clearly missing studies analyzing and presenting the potential benefits of using VWs in different game-based instructional design contexts. Highlighting the scarcity of reviews objectively aggregating a significant number of studies focused on the impact of GBL in VWs for different learning subjects, it is of great importance to conveying information in regard to the use of learning approaches and/or theoretical frameworks to understand the impact of this approach in teaching and learning. Hence, there is a reasonable need to conduct a systematic synthesis of relevant studies focusing on the use of GBL approaches in VWs, and delve into a 
critical look about the use of teaching approaches/frameworks, user experience usability issues, research methods, and students' performance in different K-12 and HE subjects.

According to the above, the current study, firstly, presents a systematic literature review of experimental studies providing qualitative and/or quantitative data to investigate course objectives and learning outcomes. Secondly, it outlines critically and systematically the effects of using GBL approaches in VWs on students' learning performance and achievements in a more accurate and up-to-date picture of the current state of literature.

\section{$2 \quad$ Research Method}

\subsection{Planning the review}

A systematic literature review was conducted by adopting the guidelines proposed by Kitchenham et al. [2007] in order to review the scientific articles, and divided as follows: a) planning, b) conducting the review, and c) reporting the review. This method entails specific steps that should be followed for the presentation of an unbiased synthesis and interpretation made by reading findings from previous works in a balanced manner. To succeed such an effort, relevant evidence that fits the pre-specified eligibility criteria could give answers in specific research questions and need to be collated the following steps.

\subsubsection{Review objectives and research questions}

The present review has a two-fold objective. The first is to provide a literature review for educators and scholars to understand the implementation of GBL aligned with instructional approaches in VWs for K-12 and HE settings. The second is to inform practitioners and instructional designers for new research activities on this topic to acknowledge any possible benefits, challenges, and drawbacks encountered by previous researchers in experimental studies. Therefore, the following research questions were formulated in order to achieve this review's objectives:

1. What are the trends using GBL in VWs?

2. What kind of instructional models and/or theoretical frameworks has been adopted?

3. Are game-based instructional approaches in VWs more effective than traditional as indicated by the existing educational research?

4. What game features and mechanics were used in prototypes to support different instructional design settings?

5. What are the benefits and challenges of using VWs in GBL?

\subsection{Conducting the review}

In this section, the main processes of conducting this review's search and the extraction of studies are outlined by identifying and searching the databases mentioned next.

\subsubsection{Search strategy}

The systematic identification of literature made by searching the most well-known databases focusing on educational technology, computer science, and social science 
topics to perform an exhaustive search. EI-Competex, ERIC, ESCBO, IEEEXplore, JSTOR, SCOPUS, ScienceDirect, Springer, Web of Science, and Wiley as the most relevant. All searches were made separately to each database. The period for the search was limited from January 2006 until December 2019, when this review was completed, because the educational use of VWs gained ground after 2006.

Additionally, a hand search of the reference lists of identified articles was undertaken. Google Scholar and the Directory of Open Access Journals as open access databases are commonly used by authors to identify any other primary sources within grey literature offering electronic access to most published literature [Norris et al., 2008]. The Google Scholar h5-index for the large category of "Engineering and Computer Science," and its separated categories related to "Computer Science" and "Educational Technology" was a good starting point. This decision was deemed as necessary since the two sub-categories are more relevant to "Education and Educational Research" and "Human-Computer Interaction" (HCI) extracted by Journal Citation Report Social Science Citation Index (JCR SSCI), and Journal Citation Report Science Citation Index (JCR SCI), respectively. Branching searches were performed by using forward and backward search procedures from the reference lists, which were consulted at the early and final stages.

To have an unbiased search strategy, two steps were taken by the authors. First, any key search started by the most popular databases, in which are provided articles related to HCI, such as Computer science/Engineering and Educational Technology. This implies that authors of this review do not focus solely on the presentation of positive results but also to some negative consequences, which may be provided by giving challenges to future works. Second, any selection bias was avoided as specific criteria were used in order to select the publications, and the authors decided the following: (a) to classify research questions and specific key terms to find in the search strategy from five different databases, (b) to propose a multistage process to extract information from articles in each stage and for each study following pre-determined inclusion and exclusion criteria, and (c) any potential inaccuracy in data extraction and misclassification did not exist as we extracted and interpreted any information provided from papers reviewed in full-text by JCR SCI and JCR SSCI lists without having any conflicts or further disagreements during the final stage of including the most relevant literature corpus of previous studies.

\subsubsection{Search criteria}

A manual search for peer-reviewed international journal articles was conducted in order to achieve the initial screening. For this review, the search terms (keywords) from any included terms related to VWs, in conjunction with several terms that could describe possible outcomes, impacts or effects by utilizing a VW- supported teaching interventions in K-12 or HE settings. To widen and combine literature searches, several techniques were utilized by this review's authors for searching key terms including the use of Boolean operators such as "OR" to identify any synonyms or " $A N D$ ” to combine any search term for each of the five research questions. The search string was composed manually in each of all databases based on the search functionality offered by each one. Table 1 below outlines the key search terms. 


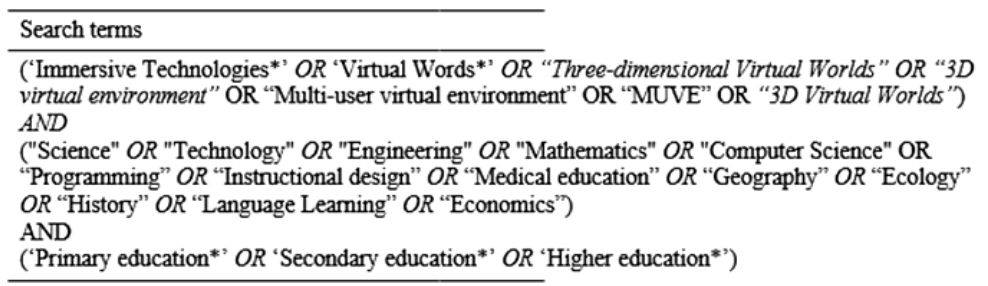

Table 1: Key search terms

Beyond the database search, any potential reviewed reference list from each included study was also checked in order to identify other relevant articles that had not been located in the database search.

\subsubsection{Inclusion and exclusion criteria}

Considering the five research questions of this review, specific inclusion and exclusion criteria for the studies to determine whether a study should be included were agreed between the two authors. The main criteria are depicted in Figure 1.

\subsubsection{Data collection}

Any relevant evidence to the pre-specified eligibility criteria that can give answers to specific research questions identified in order to be collated the following specific steps. The initial stage for data collection was guided by the principles of the PRISMA statement [Moher et al. 2009]. It is indicated by Liberati et al [2009], as one of the most appropriate protocols that authors can use to: a) report transparently strengths and weaknesses of any review investigation and b) describe adequately all eligibility criteria for study collection, information sources, data collection, and finally a synthesis of the results.

A search selection of scientific articles from five electronic databases was delivered in order to collect the most relevant studies. A total of ninety-nine articles were downloaded, organized, and entered with the aid of the StArt tool. Also, four steps based on the PRISMA statement were made to select all studies, which were finally reviewed. After identifying articles with various search procedures described above and removing duplicates, a number of sources included and excluded at each phase as depicted in Figure 2. 


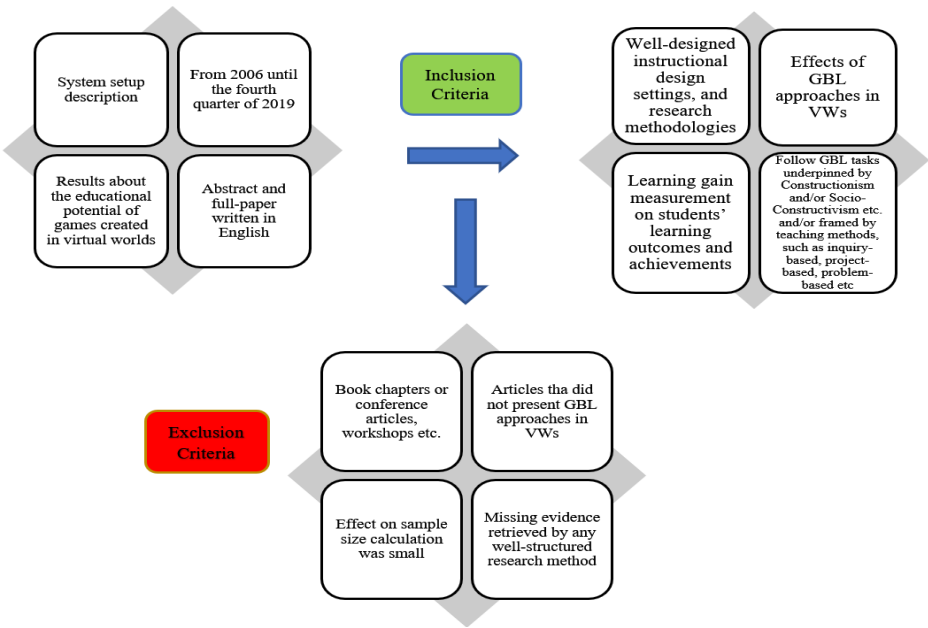

Figure 1: Inclusion and exclusion criteria

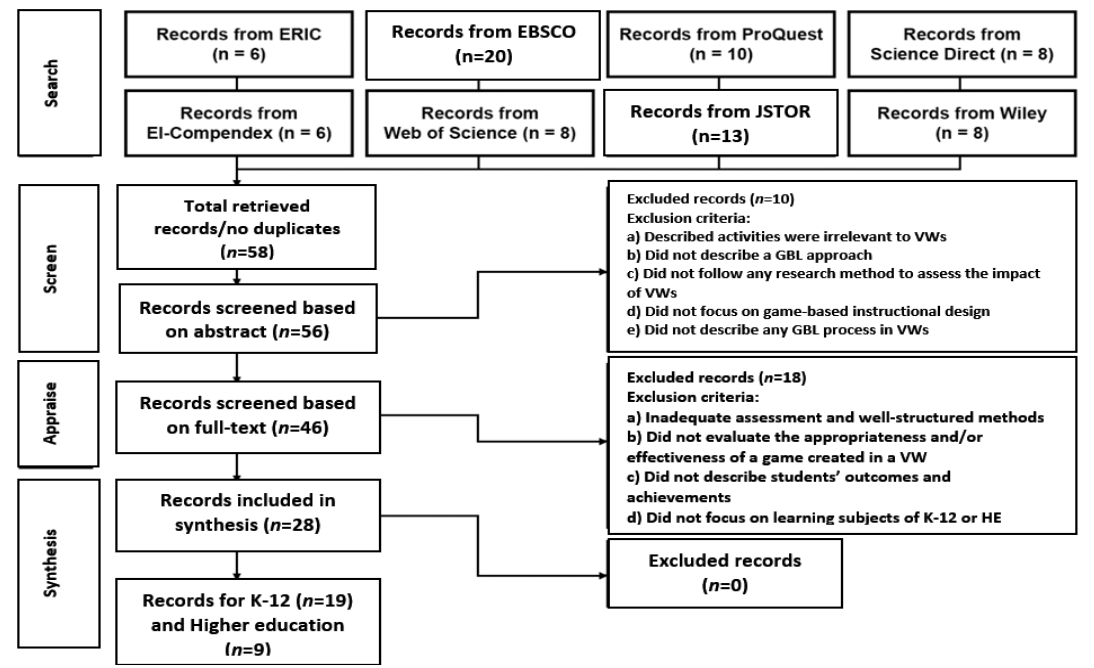

Figure 2: A flow diagram of the article selection process (adapted from Moher et al. 2009)

When the necessary searches finished and all the essential information collected by the included articles, the two authors of this review proceed with a classification of papers depending on their subject relevance and contribution to the field of educational technology. All the selected articles that were finally chosen to be reviewed had a purposeful sampling (case studies or empirical studies) based on a conscious selection of a small number of data sources. To assess the inter-rater reliability concerning the quality coding of the selected articles, a sub-sample of nineteen from a total of twentyeight articles (68\%) were included and coded independently by the two authors of this review. The inter-rater reliability $(r)$ for the total score was 0.86 , showing a good 
agreement between the authors regarding the quality of the articles that were finally reviewed.

\section{Reporting the review}

A wide range of potential benefits and challenges on $\mathrm{K}-12$ and $\mathrm{HE}$ settings were provided by the extracted data. To elaborate the discussion on the benefits and shortcomings, an aggregation of information and report a state-of-art overview from the analysis of all the included studies made to answer the main research questions.

\subsection{Trends}

The latest publication trends regarding GBL in VWs, which were published firstly from 2006 until the fourth quarter of 2019, are illustrated in Figure 3. The first articles focused on GBL in VWs were published in 2006 and 2010 in K-12 and HE, respectively. From then on, research in this area was very limited until 2006, with three publications provided until 2010.

From 2014 until 2016, GBL in VWs for different learning subjects has started gaining interest amongst scholars and education researchers, since seven $(25 \%)$ out of twenty-eight articles, in overall. Of these, four out of $19(21 \%)$ and three out of 12 studies $(25 \%)$ were applied in K-12 and HE settings, respectively.

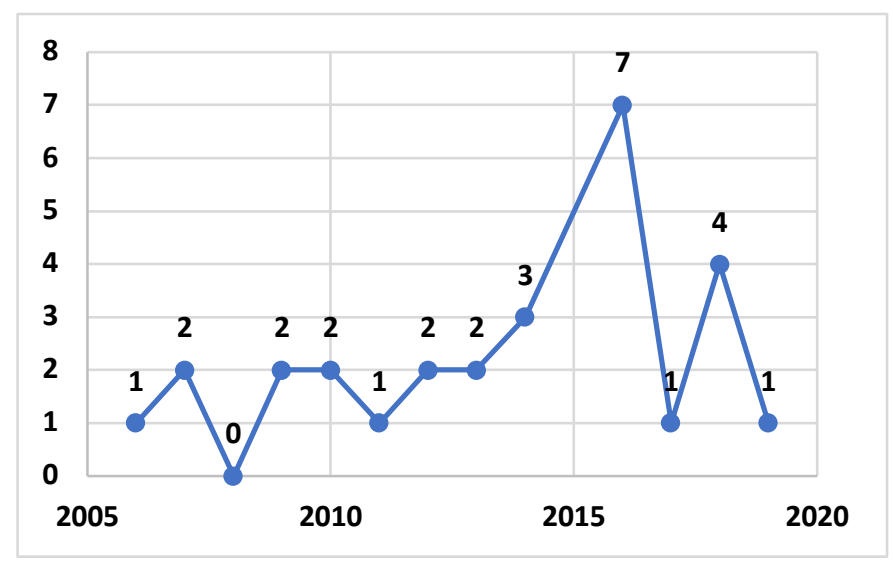

Figure 3: The number of studies published from 2000 until May 2019

This review's analysis shows that approximately $57 \%$ of the experimental research on the use of GBL approaches in VWs was published among 2014-2016. Also, an increase in the number of studies that were provided in K-12 education after 2009 and in HE after 2013, whereas a small decrease to the number of publications is observed after 2018.

Fig. 4 below reports the distribution of all the selected papers in different subject areas. While some papers referred to the use of VWs' technology, thus a subject area related to Computer Science, such studies were categorized into Instructional design 
and Technology education since participants were not engaged in coding tasks. The majority of papers in K-12 were in Science and Computer Science area with 37\% and $26 \%$, respectively. In HE, most studies were conducted in Computer Science $(22 \%)$ and Engineering (22\%) topics. A portion of the included papers in K-12 education is related to Mathematics (11\%), whereas in HE settings, some papers were conducted in Instructional Technology and Design (22\%).

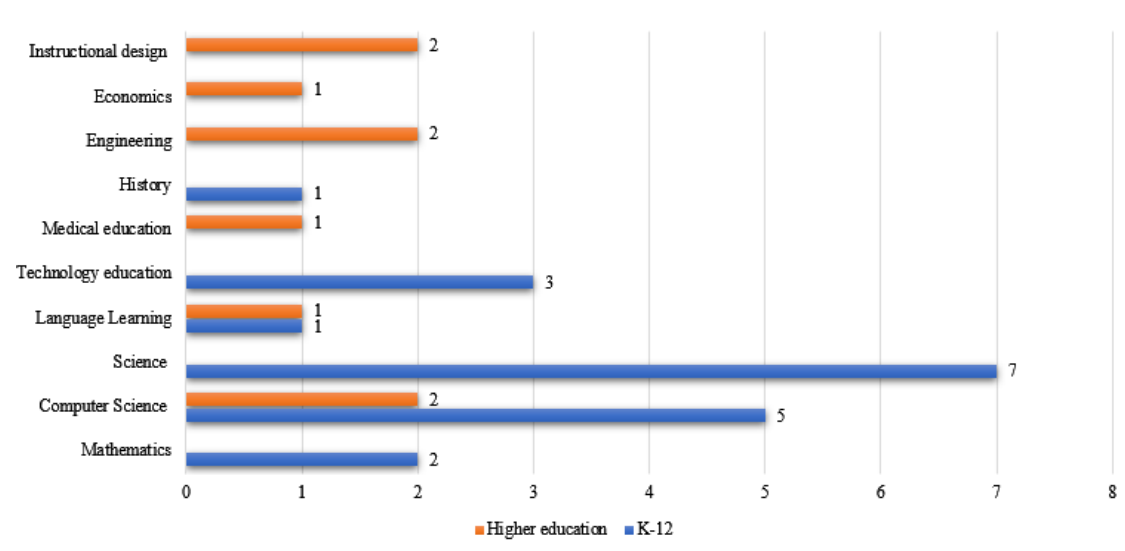

Figure 4: A summary of learning subjects in K-12 and HE settings

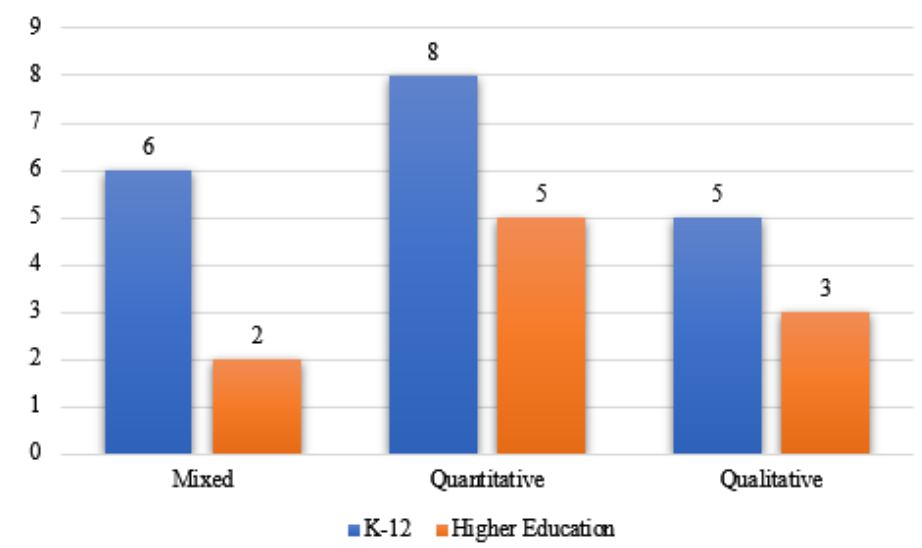

Figure 5: The research methods of the studies reviewed

Previous studies adopted various data collection methods as Fig. 5 shows. Specifically, quantitative methods in studies related to K-12 (42\%) and in HE settings $(50 \%)$ involved the comparison of learning gain, students' performance, satisfaction, user experience, usability issues, and engagement. Fewer studies used qualitative data, such as interviews, classroom recordings, and observations with $27 \%$ and $33 \%$ in K-12 and HE settings, respectively. 


\subsection{Theoretical frameworks and learning approaches}

This review's synthesis revealed that a limited number of published studies adopting a theoretical framework guiding the research and teaching practices. Out of the twentyeight scientific articles reviewed, only seven referred to an instructive-guided process framed by theoretical and/or conceptual design framework to inform about the creation of game prototypes developed in VWs. For example, Papert's theory of Constructionism emphasizes to a learning process that can become more effective when users are actively involved in a process by constructing on their something meaningful using in-game artifacts with visually-rich interactivity and behavior [Pellas 2014; Pellas $\&$ Peroutseas 2016].

The Situated learning theory allowed students to acquire professional skills by extending research on apprenticeship into how legitimate peripheral participation as being members in a community of practice [Ketelhut et al. 2007].

Embodied cognition points out a fundamental notion that thinking is directly tied to virtual bodies when students can express their feelings and communicate a/synchronously with other peers [Zheng et al. 2009; Wang et al. 2018].

Constructivism theory supports the notion of students' learning experience either with the experience that is gained in "learning by doing" or in "learning by interacting" tasks with objects of their environment in collaborative settings with their peers [Jakos \& Verber 2016].

The other three studies provided specific design guidelines for the development and creation of game prototypes in VWs. First, Barab et al. [2012] proposed the "transformational play" theory to present the responsibilities that students had by using their knowledge, skills, and concepts so as to: a) create a sense of "meaningful" situations by playing a game, b) make choices that transform the play-space within engaging tasks, and c) understand how the environment corresponds to their efforts for knowledge acquisition. Second, Pellas [2014] suggested a set of design guidelines underpinned by Constructionism for the development of a SG prototype by combining OpenSimulator and Scratch4SL palette. Third, Pellas and Vosinakis [2018] recommended a theoretical design framework for the development of simulation games.

Computer games have over time provided a significant impact on computer-assisted instruction and students' attitudes on knowledge acquisition in different scientific domains or disciplines. In alignment with game-based contexts, there are three major categories of instructional approaches to the studies reviewed by following the classification provided by Albion [2015]: Project-based learning, problem-based learning, and inquiry-based learning. Project-based learning is a student-centered approach focusing on the development of a product or a creation. Problem-based learning provides a range of tasks in which students try to subdivide a problem's parts and solve each of its parts properly to gain knowledge. Inquiry-based learning approach focuses on questioning, researching, and answering open challenging activities using students' critical and problem-solving skills (Table 1).

One of the most commonly cited rationales was the use of in-class time for "active learning" through hands-on experimental tasks rather than lecturing [Kim \& Ke 2016; Lim et al. 2006; Tüzün 2007]. 


\begin{tabular}{|c|c|c|}
\hline $\begin{array}{l}\text { Teaching methods and } \\
\text { theoretical frameworks }\end{array}$ & Reviewed studies in K-12 & Reviewed studies in $\mathrm{HE}$ \\
\hline Project -based learning & $\begin{array}{l}\text { Barab et al., 2012; Jakos \& } \\
\text { Verber, 2016; Loula et al., 2014; } \\
\text { Twining, 2009; Young et al., } \\
\text { 2012; Wang et al., } 2018\end{array}$ & $\begin{array}{l}\text { Christopoulos et al., } \\
\text { 2018; Franetovic, 2016; } \\
\text { Troconis \& Mellstrom, } \\
\text { 2010; Yilmaz \& } \\
\text { Cagiltay, 2016 }\end{array}$ \\
\hline Problem-solving learning & $\begin{array}{l}\text { Kim \& Ke, 2016; Pellas, 2014; } \\
\text { Pellas \& Peroutseas, 2016; } \\
\text { Pellas \& Vosinakis, 2018; Rico } \\
\text { et al., 2011; Şimşek, 2016; } \\
\text { Zheng et al., 2009 }\end{array}$ & $\begin{array}{l}\text { August et al., 2016; } \\
\text { Berns et al., 2013; } \\
\text { Callaghan et al., 2013; } \\
\text { Hornik \& Thornburg, } \\
\text { 2010; Granic et al., 2019 }\end{array}$ \\
\hline Inquiry-based learning & $\begin{array}{l}\text { Barab, 2005; Dede et al., 2017; } \\
\text { Ketelhut, 2007; Lim et al., 2006; } \\
\text { Metcalf et al., 2018; Tüzün, } \\
2007\end{array}$ & - \\
\hline
\end{tabular}

Table 1: Teaching methods and theoretical frameworks

Project-based learning approaches were followed broadly in K-12 Technology education [e.g. Barab et al. 2012; Twining 2009; Young et al. 2012] and learning on how its tools are utilized by someone who gain knowledge for a better disciplinary understanding. In such an approach, students encounter various challenges that they need to overcome to progress by developing and creating artifacts. They also aimed at discovering and learning how to use visual features/elements in order to apply their projects or prototypes. In-game contexts provide players the opportunity to explore and experiment with a large degree of flexibility in an "open-world". This implies the use of data, visual elements, and tools integrated within a game to engage all users within an ongoing information technology project concerning in-defined problems that are hard to define and observe in reality.

Problem-based learning approaches were mostly followed in K-12 Computer Science and Mathematics courses. The recent research literature on the field of Computer Science [Jakos \& Verber, 2016; Pellas, 2014] allowed, firstly, users to make a preliminary exploratory experience into a $3 \mathrm{D}$ environment with pre-determined tasks and interactive objects. Secondly, they should continue by learning how to program visual objects properly in order to understand how to subdivide all parts of the main problem, identify elements that can lead to an expected solution to all of its parts and then feed the first plan by starting to program. Learning how to program a scripting language supported by "social" and "open-source" VWs requires C-style syntax and specific keywords knowledge. All 3D visual elements/objects have different geometric shapes and receive several scripts that can be executed concurrently using either the programming language supported by VWs [Rico et al. 2011] or combining easily code blocks with Scratch4SL [Pellas \& Vosinakis 2018]. In Mathematics, as materials in a traditional course are delivered with difficulty, lack practicality for students' real-life in-school settings is observed, and, hence, several exercises are provided as abstract and complicated [Kim \& Ke 2016]. For instance, two studies [Kim \& Ke 2016; Şimşek, 2016] used GBL in VWs to support contextualized and active learning with the following perspectives: a) allow users to experience their mathematical practices with real-life simulated tasks, b) use 3D "anthropomorphic" digital entities to create a sense of presence to each user either by "being there" alone or in collaboration with their peers (sense of co-presence) to solve problems in practice and observe in real-time the consequences of their actions, and c) promote higher cognitive engagement with other 
avatars within 3D interactive experiences assisting users to think and comprehend complex ideas or skills easier than with traditional instruction.

As regards inquiry-based learning approaches, the results from the studies reviewed in K-12 were distributed between Life science and Social science topics. Most of the interventions related to Life science topics took place in laboratory settings, albeit language and history interventions were made in-class using desktop computers. Furthermore, Life science topics were mostly deployed using VW-supported exploratory tasks and developed tools, with inquiry activities organized by the instructor(s) to conceptualize physics phenomena. Social science topics were devoted by exploring and simulating a 3D environment, with an emphasis on the understanding of specific learning domains. As indicated by the description from previous studies, a wide range of simulation games were utilized since students had the chance: a) to play and learn various educational concepts and b) to immerse themselves into real-world simulated environments with high representational fidelity in an effort to be familiarized with intended learning concepts and materials which are difficult to be explored into real-world settings. Therefore, students seemed to learn by playing within specific role-playing contexts and observe the consequences of their actions by using theoretical knowledge to study several phenomena. They can also examine their hypotheses that can be executed in-game and reflected on their own understanding. A categorization of such simulation games can be divided into two contexts. In social science topics [e.g. Zheng et al., 2009; Wang et al., 2018], the type of exercises can be categorized as role-playing and simulation of action. In Life science [e.g. Barab et al. 2005; Dede et al. 2017; Metcalf et al. 2017], studies were focused mostly on the implementation of "drill-and-practice" tasks for hands-on tasks, the observation of simulation a system/process, and/or the simulation to improve system-based practices.

In HE settings, the studies reviewed relating to project-based $(n=7)$ and problembased $(n=5)$ learning approaches. Project-based approaches were featured predominantly not only in Computer Science [e.g. Christopoulos et al 2018; Granic et al., 2019], and Instructional Design [e.g. Franetovic 2016; Yilmaz \& Cagiltay 2016] but also in Medical education [Toro-Troconis \& Mellström 2010]. Two main GBL approaches were employed in the game making by focusing on gameplay and game development. For instance, Toro-Troconis and Mellström [2010] used a simulated health environment in Second Life, where students participated in experiential, diagnostic, and role-play learning activities related to patients' diagnoses, investigations, and treatments. On the other side, in Yilmaz and Cagiltay [2016], students developed collaboratively game-like environments for various cognitive or science topics in Quest Atlantis.

Problem-based learning approaches were also applied in multiple HE subjects, such as Engineering [August et al. 2016; Callaghan et al. 2013], Language learning [Berns et al. 2013], Economics [Hornik \& Thornburg 2010], and Computer Science [Granic et al. 2019]. In all studies, students interacted with scripted, interactive 3D objects and simulated, game-like environments developed by instructional designers. These resources were used for visualization and interaction with data [Callaghan et al. 2013], comprising highly interactive engineering demonstrations and complex simulations to assess understanding and engage users' critical thinking through active exploration [Granic et al. 2019]. These games allowed students not only to test and improve their knowledge and skills [Berns et al. 2013] but also to apply taught theoretical concepts to practical problems [August et al. 2016]. GBL activities enabled 
students to move actively and gradually from rote facts memorization to knowledge comprehension and application towards the achievement of higher-order skills [Hornik \& Thornburg 2010].

\subsection{Effectiveness of GBL approaches}

From the overall twenty-eight articles, six compared students' learning performance between traditional in-class course delivery and game prototypes developed in VWs. For instance, there were retroactive lectures between instructor(s) and students with presentations on multimedia files, such as PowerPoint slides enhanced with text, video, audio, and images, whereas others proposed the presentation of students' projects, simulation of problem-based tasks or a summary of simulation-based observations/emulations of the real-world into VWs. Nine articles were focused on students' outcomes and achievements, six on user experience measurement, and three conducted several usability tests (Table 2). Of these, seven reported improvements in average pre-and-post-test scores, and in only one study it was not reported any statistically significant difference in means between two groups.

\begin{tabular}{|l|l|l|}
\hline Major findings & Reviewed studies in K-12 & Reviewed studies in HE \\
\hline $\begin{array}{l}\text { Improvements on students' } \\
\text { learning performance }\end{array}$ & $\begin{array}{l}\text { Kim \& Ke, 2016; Jakos \& } \\
\text { Verber, 2016; Metcalf et al., } \\
\text { 2018; Pellas \& Vosinakis, 2018; } \\
\text { Şimșek, 2016 }\end{array}$ & Granic et al., 2019 \\
\hline $\begin{array}{l}\text { Increment on students' } \\
\text { outcomes and achievements }\end{array}$ & $\begin{array}{l}\text { Jakos \& Verber, 2016; Loula et } \\
\text { al., 2014; Lim et al., 2006; } \\
\text { Ketelhut, 2007; Young et al., } \\
\text { 2012; Zheng et al., 2009 }\end{array}$ & $\begin{array}{l}\text { Toro-Troconis } \\
\text { Mellstrom, 2010 }\end{array}$ \\
\hline $\begin{array}{l}\text { Gaming in VWs impact } \\
\text { positively user experience }\end{array}$ & $\begin{array}{l}\text { Pellas \& Peroutseas, 2016; } \\
\text { Simsek, 2016; Twining, 2009; }\end{array}$ & $\begin{array}{l}\text { Callaghan et al., 2013; } \\
\text { Franetovic, 2016; }\end{array}$ \\
\hline $\begin{array}{l}\text { Analysis of usability testing } \\
\text { data received students' } \\
\text { positive acceptance }\end{array}$ & $\begin{array}{l}\text { Dede et al., 2017; Granic et al., } \\
\text { 2019; Rico et al., 2011 }\end{array}$ & $\begin{array}{l}\text { August et al., 2016; } \\
\text { Berns et al., 2013; } \\
\text { Yilmaz \& Cagiltay, 2016 }\end{array}$ \\
\hline $\begin{array}{l}\text { Enhancement on students' } \\
\text { engagement and active } \\
\text { participation }\end{array}$ & $\begin{array}{l}\text { Barab et al., 2012; Pellas, 2014; } \\
\text { Tüzün, 2007; Wang et al., 2018 }\end{array}$ & $\begin{array}{l}\text { Christopoulos et al., } \\
\text { 2018; Hornik } \\
\text { Thornburg, 2010; }\end{array}$ \\
\hline
\end{tabular}

Table 2: Findings from the studies reviewed

However, research findings reflected on the impact of GBL in VWs to measure student learning outcomes, as well as their performance, remain mixed. In K-12 education, Barab et al. [2012] argued that students who assigned in game-based units created in Quest Atlantis reported significantly higher levels of engagement and had fewer reprimands from their teacher in terms of completing specific goals than those with story-based tasks. Jakos and Verber [2016] advocated that GBL in OpenSimulator assisted students to reach successfully a minimum standard of knowledge by playing only the educational game in introductory programming as provided by the pre-andposttests. Kim and Ke [2016] urged on the use of OpenSimulator in Mathematics since the treatment group had a positive effect in problem-solving scenarios with better math learning achievements than those who followed a traditional (face-to-face) teaching approach. Pellas and Vosinakis [2018] examined the learning gain on students' computational thinking skills. The results showed that students who did not have a good 
background in programming seemed to benefit using the combination of Scratch4SL with OpenSimulator as they performed significantly better by applying their computational problem-solving strategies both in measures of problem-solving and algorithmic thinking than those who utilized Scratch. Şimşek [2016] reported an important effect of Second Life on students' attitudes towards mathematics and improvements in the duration of study hours. Lastly, Metcalf et al. [2018] reported that the average of students' final scores between those who utilized paper-based formats and their counterparts who used an electronic concept mapping tool. The same authors found that an electronic concept-mapping tool had significant advantages in supporting the construction of concept maps representing, conceptual understanding and causal relationships in a VW-supported system than their peers who used traditional paperand-pencil approaches.

Other studies in K-12 were focused on user experience and game usability measurement. Some of the most indicative positive results are as follows: a) integration of science inquiry tasks in interactive VWs improved students' self-efficacy and engagement [Ketelhut 2007], b) game development in VWs seemed to be a good vehicle for providing people with "lived experiences" allowing students to overcome possible restrictions that are difficult or impossible to do in the physical world [Twining 2009], c) combination of the effectiveness of "constructionist-oriented" instructional settings for students' engagement to acquire or empower social, cognitive, higherorder, and computational thinking skills, d) attention and energy expenditure of students to complete certain in-game tasks (behavioral engagement) had not only a linear correlation with learning strategies for knowledge acquisition (cognitive engagement), but it had also a positive association with their emotions and achievements/outcomes (emotional engagement) in collaborative learning tasks [Pellas 2014], and e) a 3D game to teach social competence due to the spatial and visual technologically-rich features of VWs alongside with a-/synchronous communication tools can support collaborative learning of students with autism spectrum disorder [Wang et al. 2018].

In HE settings, August et al. [2016] found that students who used Second Life in cross-disciplinary STEM subject areas, such as positional numbering systems, logical operations, circuit design, and differential equations, performed higher as indicated the perspective from users' experience than their counterparts who followed a traditional classroom delivery with lecture-based sections. Despite both groups started with similar levels of prior knowledge and improved further their learning performance, the Second Life-supported group improved their scores than their counterparts, there was not found any statistically significant difference in favor of students' learning improvements. In their study, Christopoulos et al. [2018] investigated the influence of educational games on students' understanding with regard to the learning material and learning acquisition. The same authors noticed that GBL in VWs allows students to interact with their peers and with in-world visual objects; thus, they provide as well as overall enhanced levels of engagement and learning acquisition. The results from Granic et al.'s [2019] study indicated that interface usability in VWs influenced positively user experience as provided by memorability tests about information security after a longer period of inactivity of OpenSimulator's visual objects. The assessment showed a statistically significant difference in students' learning performance through the measure of acquired knowledge after completing two scenarios in the VW. 


\subsection{Game features and mechanics}

Researchers proposed a wide range of game features and mechanics as essential parts of games. According to Prensky [2007], six key structural game features need to be considered for in-game tasks: (a) rules; (b) goals; (c) outcomes and feedback; (d) challenges; (e) interaction; and (f) story. Such features are observed as game mechanics and provided as follows. Challenges are the missions that players will face and try to achieve each in-game goals using acquired knowledge aligned with practical and/or cognitive thinking skills. A specific storyline is a set of steps that describes a problem statement that players would face by playing a game. The immediate rewards will be received when players accomplish certain tasks related to challenges and in-game goals. Lastly, the integration of game-play is aligned with the provided learning content using visual objects and elements [Kim \& Ke 2016].

In Science education, Tüzün [2007] proposed a game, in which primary education students experienced a problem-based scenario allowing them to control non-playable characters (NPCs) that were lost in a game world based on the information and clues provided in the environment. In secondary education, Loula et al. [2014] gave students several opportunities to infer knowledge by interacting with 3D digital content representing visual elements, such as simulation and puzzles to provide immediate feedback on their actions through instructive guided conditions. In another study, Barab et al. [2012] proposed "transformational play" as a theory to describe the potentials positioning of a player's character in order to understand better (role-playing) gamebased contexts in two ways: a) by playing a game and taking a specific role of a protagonist who must employ conceptual understandings, and b) by making specific choices that have sense to investigate the reasons and rationale behind a mystery to solve. Such a context provides small story-based units design that students are engaged by learning relevant concepts and skills, while simultaneously create a context, in which they can practice and learn by evolving their understandings on a specific topic.

In Computer Science, Jakos and Verber [2016] developed a game named "Aladdin and his flying carpet'. It was a fantasy game that described the traveled path of the object on a virtual grid. Students are not only challenged to propose a solution by "drilland-practice" but also to learn how to program in pseudocode. Pellas and Vosinakis [2018] developed a "robot vacuum cleaner" simulator using OpenSimulator combined with Scratch4SL and in Scratch for secondary education curriculum. House furniture and objects in square floors were seen as evocative spatial metaphors of basic geometric shapes (e.g., triangle, square, and hexagon), making players think and practice computationally with an abstract conceptualization approach. Second, they needed to program pathfinding in a logical problem to traverse an autonomous robot vacuum cleaner so as to execute specific cleaning pathways, without causing any damages.

In Mathematics, Kim and Ke [2016] developed a simulation game in OpenSimulator, in which elementary school students prepared a picnic for four friends. The main goal was for each user to spend less amount of money buying food and complete four challenges to get his/her best friend's meals. Player rewards were announced, when each challenge was accomplished by spending as less virtual money as possible. Şimşek [2016] developed a 3D mathematic robot created in Second Life to support informal instruction, in which secondary school students had as the main objective to explain the 2D Cartesian system's coordination comprised by visual objects. 
In HE settings, two main GBL integration modes were observed; (i) games of short duration in a supportive role to a wider learning scenario and (ii) complex games with multiple levels and mechanisms that constitute the entirety of the learning experience. Supportive learning games were deployed creatively and effectively in Engineering [e.g. August et al. 2016], Economics [e.g. Hornik \& Thornburg 2010], and Computer Science [e.g. Christopoulos et al Educators chose specific difficult abstract concepts and skills and created interactive 3D objects to generate several challenges for students' skills cultivation and provide immediate feedback. Such games were played in single mode taking the form of traditional games, like crosswords, slot machines or anagram puzzles. The interaction with them was realized mainly through chat messages or buttons. Hornik and Thornburg [2010] used an innovative mode in one of their supportive games combining role play by making the student avatars' game components; the students became actual classifiable pieces in an accounting game to practice their understanding. In all these instances, the developed games, even though they held a supplementary, secondary role in learning, their availability to students lead to increased engagement. Callaghan et al. [2013] took another notable step towards proposing a sophisticated integration of game prototypes learning environments by connecting them with the institutional Learning Management System (LMS), and by adding interoperability with further hardware in the physical world, such as Wii mote.

On the other hand, educators with clear learning objectives and intended outcomes were able to design and build learning experiences combining a variety of complex game mechanics across different subjects such as Computer Science [Granic et al. 2019], Language learning [Berns et al. 2013], Instructional design [Yilmaz \& Cagiltay 2016] and Medicine [Toro-Troconis \& Mellström 2010]. On the technical side, most of these games used a 3D VW to provide interaction and feedback. Toro-Troconis and Mellström [2010] built an elaborate simulation using various patient data to help medical students practice and apply their knowledge. Granic et al. [2019] organized simulated scenarios with interactive quizzes and personalized educational materials to train students on cybersecurity. Yilmaz and Cagiltay [2016] challenged students to design and create single-user games that featured learner control, competition, and story. Berns et al. [2013] created a simulated game-based environment consisting of multiple levels in the form of rooms that were linked with each other. Each level prepared students for specific sub-skillset. Some of the levels used an important affordance of VWs that is social interaction in the form of team competition. Students within such game-based contexts were allowed to play the final level only if they achieved the minimum set results in previous levels.

\subsection{Benefits and challenges}

Game-based learning in VWs provides not only benefits but also challenges. According to previous studies' findings, games that developed in VWs provide user interface design features and elements with realistic simulated representational fidelity by displaying a 3D digital environment. In specific, such a process allows the observation and execution of users' actions so as to assess the consequent results of those actions in simulated problem-solving tasks using as well as a/-synchronous communication, i.e. situations that resembled similarly as those to the real world by taking advantage of intuitive, natural modality contexts for user-interaction tasks. Such learning contexts 
lead to students' outcomes/achievement improvements, and enhanced levels of participation and engagement in learning subjects. Within instructive-guided GBL settings, students had several opportunities to acquire a set of cognitive skills related to higher-order [Ketelhut 2007], algorithmic and computational thinking [Pellas \& Vosinakis 2018], critical thinking [Lim et al. 2006], and problem-solving [Şimşek 2016] in realistic simulated several tasks with high representational fidelity.

VWs provide a set of interactive visual metaphors and elements that students can use for training and practice to replicate a wide range of (realistic) learning scenarios for problem-solving, project-based and inquiry-based learning tasks. To be achieved properly such approaches, the instructor(s) should observe and communicate spatially with all students and provide the appropriate feedback in real-time. Such an approach additionally needs well-designed instructional contexts [Franetovic et al. 2016; Yilmaz \& Cagiltay 2016]. For other studies [Zheng et al. 2019; Wang et al. 2018], "embodied social presence" with avatars was one crucial factor behind the use of GBL for spending students' time and efforts to more complex exercises meaningfully both inclass and online instructional settings.

Another aspect was the correlation between student engagement and knowledge. The current review's synthesis revealed an increased level of students' engagement in problem-solving tasks [Hornik \& Thornburg, 2010; Pellas, 2014]. Other studies found that students achieved better outcomes under the guidance of their instructor(s) following either project-based learning [Wang et al., 2018] or problem-based learning exercises [Barab et al., 2012; Zheng et al., 2009]. Christopoulos et al. [2018] admitted that interactions among students by playing games in VWs led to higher levels of engagement, and thence to a better learning acquisition. However, the interactions among students hosted in OpenSimulator were weaker, compared with the ones observed in the physical classroom.

In contrast, Pellas [2014] pointed out significant demarcation between knowledge acquisition and engagement factors indicating that any different type of interactions in VWs aimed at providing students with interactive and problem-based learning activities do not approximately lead to a better understanding of the learning material as well as knowledge acquisition. Even though the use of GBL in VWs can influence positively the behavioral, cognitive and emotional engagement of students due to high levels of immersion, this cannot guarantee either that students can complete properly a range of problem-solving tasks in terms of having good academic performance and course grades or that their interactions with their peers or with in-game visual objects will lead to higher level of knowledge acquisition.

As with any new instructional design approach, GBL in VWs brings crucial challenges for instructors and students. The major challenges for instructors were some technical issues in order to apply course delivery methods in-class or online. Instructional design development requires both a reasonable time effort and support since in-class there is always one instructor, but online, there should be a common time presence of all members [Yilmaz \& Cagiltay 2016]. Challenges, especially for younger students, include technical equipment issues, Internet security, and knowledge about online (supplementary) approaches could be insufficient with curriculum-aligned issues. For example, in Barab et al.'s [2012] study, students felt to be lacked their meaningful engagement in long time tasks without reasonable objectives. Other challenges which may restrict the use of VWs are technical issues, e.g. "freezing" or lag issues, lack of opportunities for ready-made tools and game authoring tools can 
cause hindrance at their first-time entry, use increased workload for game designers and cause students' boredom or frustration [Pellas \& Peroutseas 2016; Yilmaz \& Cagiltay 2016]. Also, Granic et al. [2019] mentioned several shortcomings related to system technical issues, like slow feedback, frequent lags, poor graphics, and appropriateness of the VW regarding any complicated movement as well as some inappropriateness of the interface for younger children (e.g., naked avatars). Other researchers [Zheng et al. 2009; Young et al. 2012] found that students could easily skip by using in-game learning materials and tended to make their modifications on avatars or other in-game elements/objects. This may cause failure to students who want to finish exercises on their own and then collaborate with other peers so as to accomplish in-game objectives. Although the findings in terms of class attendance varied, some researchers found that GBL increased participation and limited failure on students' achievements and outcomes. For instance, Okutsu et al. [2013] found that $95 \%$ of students gained more confidence to demonstrate their achievements. Likewise, Kim and Ke [2016] found out that students logged into the VW supported platform more frequently in order to exercise their practical skills and access course materials compared to the face-to-face in-class teaching approach.

Notwithstanding students are "digital natives" as they were ubiquitously and daily utilizing several technological-advanced devices and applications, there are several concerns implied that technical issues irritated them to keep alive the "learning momentum". The lack of control over the given tasks or the superficial prior familiarity of students with VWs' objects/items may prevent them from paying attention to particular tasks or getting real-time feedback alongside limited modifications from items, such as sound or gestures. Such permissions can largely restrict the way that game developers and instructors can also develop or integrate learning materials into "social" VWs. An alternative option to have fully controlled-access of avatars' tasks and communication channels is the use of "open-source" VWs [August et al. 2016]. Moreover, due to the lack of game authoring tools, game designers and developers should consider how to mix properly visual and auditory stimuli so as to assist students without having any negative effect depending on the complexity of learning concepts that can eventually increase the germane cognitive load [Jakos \& Verber 2016].

Students' resistance was another crucial challenge as revealed by previous studies. GBL in VWs made sometimes students feel overwhelmed, especially on their involvement to assimilate new instructional approaches for active participation in different learning tasks [Hornik \& Thornburg 2010; Ketelhut 2007]. Also, students may find time-consuming the fact that they need to learn a high-level programming language to use LSL, thus they tend to develop and program small projects. However, the use of Scratch4SL within well-defined instructional contexts can prevent such frustrating processes [Pellas 2014].

\section{Discussion}

The publication trends indicate that there is an increasing interest in using VWs to a wide range of learning subjects in K-12 and HE settings, although a gradual decrease of publications in peer-reviewed journals identified during the last three years. Nevertheless, the scarcity of experiments with long-term and large sample-size in journal publications shows that research on GBL in VWs requires more effort to be 
made. Many studies adopted their practices in VWs through simulation-based games, and focused on documenting game usability and engagement processes, while some others shared evidence based on user experience focusing on preliminary results from students' opinions and impressions.

Theoretical design frameworks assisted educators/instructors to create a good statement about the significance of in-game problems and organize properly the selection of appropriate learning materials and pave a pathway for knowledge acquisition [Pellas \& Peroutseas 2016]. Nonetheless, there is a limited number of studies adopting theoretical and/or conceptual design frameworks guiding the development and evaluation of game prototypes that can be developed in VWs. If GBL has the potential to be aligned with learning theories/theoretical underpinnings, such as Constructionism [Pellas 2014], Embodied Cognition [Zheng et al. 2014], and Socioconstructivism [Ketelhut et al 2007], then future researches on the design and development of games in VWs need to provide more details on how such frameworks or models can be applied successfully. Therefore, theoretical design models can provide practices and learning approaches in GBL contexts for designing, developing and implementing instruction in VWs. The use of theoretical underpinnings constitutes a wide range of specific steps that students should follow under the guidance of their instructor(s) within (task-based) learning scenarios as a sequence of typical tasks and actions for knowledge acquisition.

One of the most significant aspects behind converting a course from a traditional format to GBL was the use of class meetings for "learning-by-doing" tasks rather than for lecturing. Nonetheless, active learning itself is not enough to be proposed and applied differently by researchers and educators. For instance, a multitude of activities without being guided by any instructor(s) can have unexpected results, and thence to not assist students' knowledge acquisition when compared to traditional sessions. The use of VWs cannot always offer positive results on students' learning performance when, for example, courses related to second language instruction require more preferably the use of LMS due to the ready-made authoring tools which are provided [Berns et al. 2013]. Therefore, specific pedagogical models and theoretical underpinnings that have as an axis a range of "learning-by-doing" tasks, such as problem-based learning and project-based learning could provide clear benefits for educators and students in different learning subjects [Dede et al. 2017; Ketelhut 2007; Pellas \& Peroutseas 2016].

The current systematic literature review is in line with previous ones [Pellas et al., 2017; Wang \& Burton 2013], which provided several perspectives from the implementation of more experimental studies so as to investigate the effectiveness of GBL. Beyond the fact that many studies presented evidence based on various assessment research methods, this review unveils that such methods were limited to student engagement, measurement of learning gain and surveys getting user experience feedback. Beyond the fact that such methods can give valuable information about the role of GBL in VWs; however, they may fall short in analyzing the overall impact on students' learning performance.

To summarize, the results gathered and analyzed from comparative studies indicated that learning in VWs was more effective in terms of achievements/outcomes than their counterparts who followed traditional approaches. A remarkable option to strengthen such an opinion could be to conduct a meta-analysis so that make a definitive conclusion about the use of GBL in VWs over the traditional ones. The vast majority 
of studies reported analytically mean scores, standard deviations based on different measurements (e.g., final course grades, quiz scores, project creations, etc.), and a wide number of observations or interviews. Nonetheless, it is of great importance to mention that even if a game provides enjoyable and playful contexts, it does not necessarily mean that it will lead students to have better learning gain.

\section{$5 \quad$ Recommendations and implications}

There is good evidence from previous studies indicating that the use of interactive games developed in VWs can significantly influence students' engagement and knowledge acquisition in different learning subjects and instructional tasks. Yet, alongside several comparative studies to measure the effectiveness of GBL approaches in contrast to traditional ones, others were focused on measuring user experience or usability issues of games developed in VWs. Nonetheless, further analysis in regard to the transferability of these findings to different learning contexts is required. Specifically, any claims about the tools that students have already know how to use in previous tasks and can be combined with VWs. For example, some studies [Loula et al. 2014; Pellas, 2014; Toro-Troconis \& Mellström 2010] analyzed the way that students interacted with their peers in problem-solving sessions using synchronous communication tools and shifted their conversations from simply recalling facts to conceptual discussions in order to achieve in-game objectives.

Some challenges from the studies reviewed were mainly for younger students who do not have the appropriate background and technical experience, such as heavy workload to learn the programming language of VWs can make this technology frustrating for the development of games in VWs. A possible solution, which can be addressed effectively, is the combination of VWs with Scratch4SL. It is a visual palette with colored code blocks that allow students to code any visual object into a VW. Instructors might be advised to gradually convert their courses in formal or (supplementary) informal contexts rather than keeping students in-class to learn how to code causing also a sense of boredom and disengagement with repetitive lectures [Pellas \& Vosinakis 2018]. A second one that can assist instructor(s) to measure student performance in a game-based environment is the combination of VWs with 2D platforms, such as Sloodle (Simulation Linked Object-Oriented Dynamic Learning Environment). It is an open-source e-learning software project that gives instructional designers and instructor(s) the chance to combine several tools which are already known using Sloodle and facilitate them to exchange data between the two environments. For instance, a game prototype in VW can become the main platform for the implementation of experiential and exploratory learning tasks with interactive demonstrations and simulations that can be combined with Sloodle tools as a means for recording user actions/interactions, detecting and/or recording interactions among users with other peers or with in-VW objects and elements to gain information related to their learning experience or outcomes [Callaghan et al. 2013; Granic et al. 2019].

From the analysis of previous studies, a lack of theoretical design frameworks or models identified to propose specific guidelines for designing and developing games in VWs. This may impact negatively instructional designers and game developers who want to follow specific design guidelines either to create the same or to integrate some new elements and content into already known games that are presented for various 
educational purposes [Pellas et al. 2017; Yilmaz \& Cagiltay 2016]. Interactive visual metaphors and objects/elements in VWs should be carefully designed to provide problem-solving tasks, allowing students to apply their cognitive thinking strategies by giving alternative and acceptable solutions to various problems with different levels of difficulty. This would increase students' participation, learning gain, and engagement even in collaborative settings so as to interact with others and the instructor(s), and finally to be assigned in-class and/or online sessions as concluded by the recent literature [Christopoulos et al. 2018; Ketelhut et al 2007].

The synthesis from the existing research findings and limitations, as Figure 6 shows, advances the knowledge by bringing several implications to design and develop games in VWs. Such an approach can give a list of design guidelines for successful applications in courses helping policymakers and practitioners to clarify different types of problems and propose solutions that could give valuable answers. Therefore, the concept of proposing design recommendations is crucial, as challenges and limitations on research from the previous literature focused mostly on user experience and outcomes on GBL contexts supported by VWs.

\section{Conclusion}

This systematic review is timely as various games in VWs have been proposed in different learning subjects. It is of great importance to understand the current practices so as to shed light on future implementations. In this review, twenty-eight articles included and framed by five major research questions. First, the findings indicated a widespread adoption of GBL in the majority of subjects. Second, there is a scarcity of studies reporting any instructive-guided theoretical frameworks. Additionally, assessment methods have been mostly limited to quantitative data drawn from comparative and user experience studies, and there is a lack of conducting large-scale (longitudinal) qualitative research to understand phenomena in-depth within GBL contexts. 


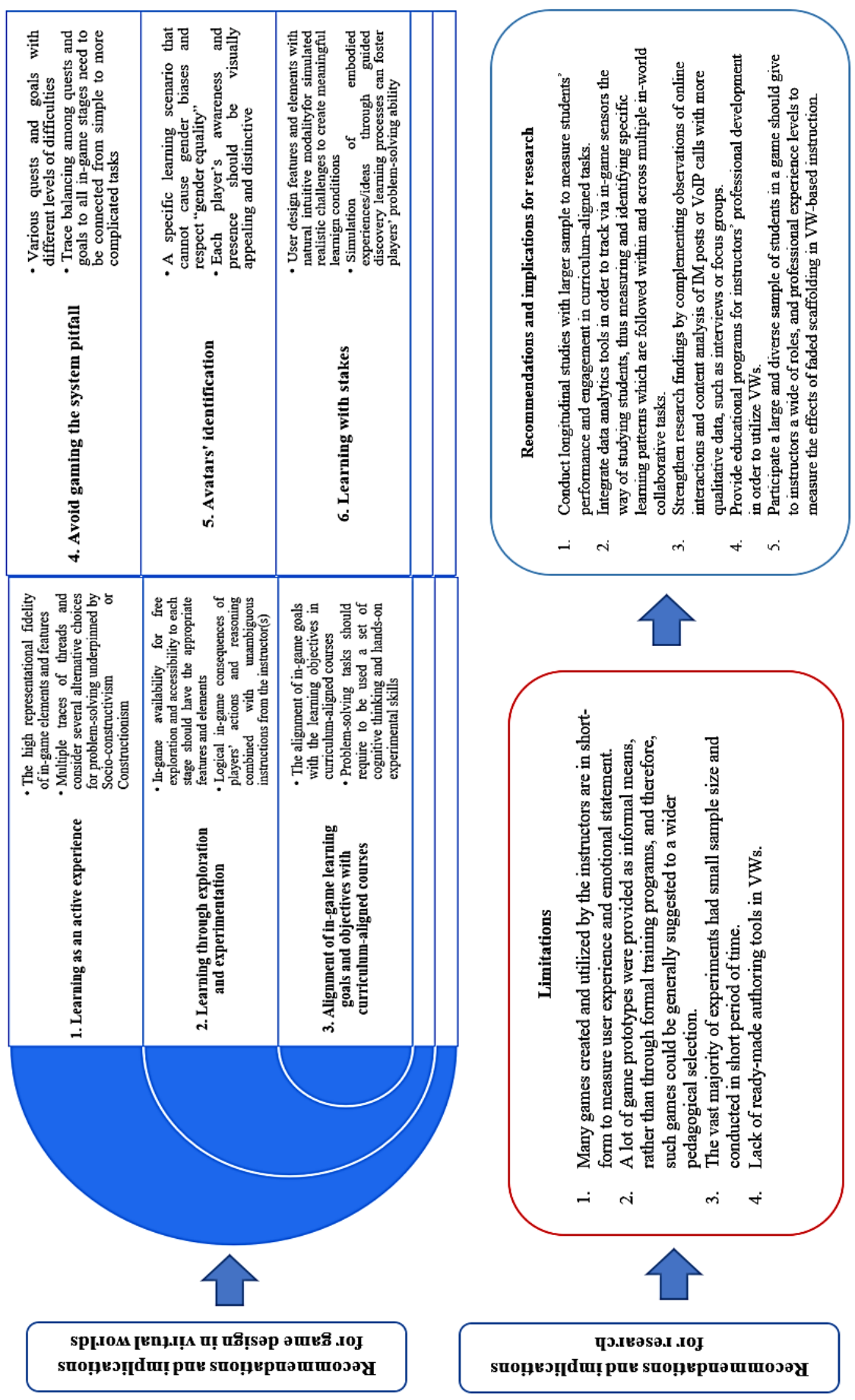

Figure 6: Recommendations and implications for game design practice and policy 
For instance, the measurement of students' engagement and performance in long-term either in formal or informal contexts to assist educators and instructors needs further investigation without any hesitation about individual contributions of already known tools that can be utilized in align with VWs. Third, a large body of literature admitted that students who followed GBL in VWs had successfully achieved better outcomes than their counterparts in traditional (lecture-style) formats.

To conclude, the contribution of this review is threefold. First, it summarizes the best pieces of advice and suggestions about game-based instructional design processes and practices in VWs. Second, it offers to researchers several insights regarding the impact of using games in VWs on different learning subjects in K-12 and HE settings. Third, it provides evidence on how well-designed activities in game-based contexts can potentially increase students' engagement, performance, and self-efficacy.

\section{$7 \quad$ Limitations and Future Work}

There are several limitations that should be noticed. First, the search selection criteria and methodology to gather all the included articles so as to consider only those published by international peer-reviewed journals decreases the total number of articles. Second, as many presentation types and topics for conferences on VW supported teaching and learning as well as those from published books can vary from the journals' perspectives, the results might have slightly differed, when considering such articles in this review. This review's findings are restricted by focusing solely on the use of VWs in education, and not, for example, the general scope of using Virtual Reality, and thence, the results provided different insights and challenges. Third, all the included articles were written only in English and any other studies written in other languages which may provide other challenges and benefits cannot be analyzed. Fourth, this review is not exhaustive, since there are appeared some databases, which do not provide the possibility to access all full-texts without payment, such as IGI Global. Consequently, some studies could not be found and analyzed.

Future work should conduct, firstly, some controlled mixed-method experiments in long term with larger sample sizes, such as longitudinal to investigate the efficacy of GBL in VWs. Secondly, in-game data analytics and tracking methods with integrated NPCs or external electronic devices to measure students' learning performance need further investigation.

\section{References}

[Albion 2015] Albion, P. "Project-, problem-, and inquiry-based learning". In Henderson, M. (eds.): Teaching and digital technologies: big issues and critical questions. Port Melbourne: Cambridge University Press, (2015), 240-252.

[August, et al. 2016] August, S.E. et al. "Virtual engineering sciences learning lab: Giving STEM education a Second Life". IEEE Transactions on Learning Technologies, 9, 1 (2016), 18-30.

[Barab et al. 2005] Barab, S. A., Thomas, M. K., Dodge, T., Carteaux, B., \& Tuzun, H. "Making learning fun: Quest Atlantis, a game without guns". Educational Technology Research and Development, 53, 1 (2005), 86-107. 
[Barab et al., 2012] Barab, S. et al. "Game-based curriculum and transformational play: Designing to meaningfully positioning person, content, and context”. Computers \& Education, 58, 1 (2012), 518533 .

[Berns et al. 2013] Berns, A., Gonzalez-Pardo, A., \& Camacho, D. "Game-like language learning in 3-D virtual environments”. Computers \& Education, 60, 1 (2013), 210-220.

[Callaghan et al. 2013] Callaghan, M. et al. "Using game-based learning in virtual worlds to teach electronic and electrical engineering". IEEE Transactions on Industrial Informatics, 9, 1 (2013), 575584 .

[Christopoulos et al. 2018] Christopoulos, A., Conrad, M. \& Shukla, M. "Interactions with educational games in hybrid virtual worlds". Journal of Educational Technology Systems, 46, 4 (2018), 385-413.

[Dede et al. 2017] Dede, C., Grotzer, T. A., Kamarainen, A. \& Metcalf, S. "EcoXPT: Designing for Deeper Learning through Experimentation in an Immersive Virtual Ecosystem". Educational Technology \& Society, 20, 4 (2017), 166-178.

[Jakos \& Verber, 2016] Jakos, F. \& Verber, D. "Learning basic programming skills with educational games: A case of primary schools in Slovenia". Journal of Educational Computing Research, 55, 6 (2016), 673-698.

[Franetovic 2016] Franetovic, M. "Future game developers within a virtual world: Learner archetypes and team leader attributes". Journal of Educational Multimedia and Hypermedia, 25, 4 (2016), 343361.

[Hew \& Cheung 2010] Hew, K. \& Cheung, W. "Use of three-dimensional (3-D) immersive virtual worlds in K-12 and higher education settings: a review of the research". British Journal of Educational Technology, 41, 5 (2010), 33-55.

[Hornik \& Thornburg 2010] Hornik, S. \& Thornburg, S. "Really engaging accounting: Second Life TM as a learning platform". Issues in accounting education, 25, 3 (2010), 361-378.

[Gee 2007] Gee, J. P. "Good video games and good learning: Collected essays on video games, learning, and literacy”. USA, New York: Peter Lang, 2007.

[Ghanbarzadeh \& Ghapanchi 2018] Ghanbarzadeh, R. \& Ghapanchi, A. H. "Investigating various application areas of three-dimensional virtual worlds for higher education". British Journal of Educational Technology, 49, 3 (2018), $370-384$.

[Girvan 2018] Girvan, C. "What is a virtual world? Definition and classification". Education Technology Research \& Development, 66, 5 (2018), 1087-1100.

[Granic et al. 2019] Granic, A., Nakic J. \& Marangunic, N. "Scenario-based group usability testing as a mixed methods approach to the evaluation of three-dimensional virtual learning environments". Journal of Educational Computing Research. DOI: 10.1177/0735633119859918, 2019.

[Ketelhut 2007] Ketelhut, D. J. "The impact of student self-efficacy on scientific inquiry skills: an exploratory investigation in river city, a multi-user virtual environment". Journal of Science Education and Technology, 16, 1 (2007), 99-111.

[Kim and Ke] Kim, H. \& Ke, F. "Effects of game-based learning in an OpenSim-supported virtual environment on mathematical performance". Interactive Learning Environments, 25, 4 (2016), 543555 .

[Kitchenham et al. 2007] Kitchenham, B. et al. "Guidelines for performing systematic literature reviews in software engineering (Version 2.3)". EBSE Technical Report, Keele University and University of Durham. Retrieved 15 October 2019 from https://www.elsevier.com/_data/promis_misc/525444systematicreviewsguide.pdf, 2007. 
[Liberati et al. 2009] Liberati, A. et al. "The PRISMA statement for reporting systematic reviews and meta-analyses of studies that evaluate healthcare interventions: Explanation and elaboration". BMJ, 339: b2700, 2009.

[Lim et al. 2006] Lim, C. P., Nonis, D. \& Hedberg, J. G. "Gaming in a 3D multiuser virtual environment: engaging students in Science lessons". British Journal of Educational Technology, 37, 2 (2006), 211-231.

[Loula et al. 2014] Loula, A. et al. "Modeling a virtual world for the educational game Calangos". International Journal of Computer Games Technology, 2, 3 (2014), 1-14.

[Metcalf et al. 2018] Metcalf, J., et al. "Supports for deeper learning of inquiry-based ecosystem science in virtual environments - Comparing virtual and physical concept mapping". Computers in Human Behavior, 87, 5 (2018), 459-469.

[Moher et al., 2009] Moher D, Liberati A, Tetzlaff J, Altman DG. "The PRISMA Group Preferred Reporting Items for Systematic Reviews and Meta-Analyses: The PRISMA Statement". PLoS Med 6(7): e1000097. DOI: 10.1371/journal.pmed1000097, 2009.

[Mystakidis and Berki 2018] Mystakidis, S., \& Berki, E. "The case of literacy motivation: Playful 3D immersive learning environments and problem-focused education for blended digital storytelling". International Journal of Web-Based Learning and Teaching Technologies, 13, 1 (2018), 64-79.

[Mystakidis et al. 2019] Mystakidis, S., Cachafeiro, E., \& Hatzilygeroudis, I. "Enter the Serious E-scape Room: A Cost-Effective Serious Game Model for Deep and Meaningful E-learning". In Proceedings of the 10th International Conference on Information, Intelligence, Systems and Applications. Patras, 15-17 July 2019: IEEE, 2019.

[Norris et al. 2008] Norris, M., Oppenheim, C., \& Rowland, F. "Finding open access articles using Google, Google Scholar, OAIster and OpenDOAR". Online Information Review, 32, 6 (2008), 709.

[Pellas 2014] Pellas, N. "Exploring interrelationships among high school students' engagement factors in introductory programming courses via a 3D multi-user serious game created in OpenSim". Journal of Universal Computer Science (J.UCS), 20, 12 (2014), 1608-1628.

[Pellas and Peroutseas 2016] Pellas, N. \& Peroutseas, E. "Gaming in Second Life via Scratch4SL: Engaging high school students in programming courses". Journal of Educational Computing Research, 54, 1 (2016), 108-143.

[Pellas et al. 2017] Pellas, N., Konstantinou, N., Kazanidis, I. \& Georgiou, G. "Exploring the educational potential of three-dimensional multi-user virtual worlds for STEM education: A mixed-method systematic literature review". Education \& Information Technologies, 22, 5 (2017), 2235-2279.

[Pellas and Vosinakis 2018] Pellas, N. \& Vosinakis, S. “The effect of computer simulation games on learning introductory programming: A comparative study on high school students' learning performance by assessing computational problem-solving strategies". Education \& Information Technologies, 23, 6 (2018), 24232452.

[Prensky 2007] Prensky, M. "Digital game-based learning”. USA: Paragon House, 2007.

[Rico et al. 2011] Rico, M. et al. "Improving the programming experience of high school students by means of virtual worlds". International Journal of Engineering Education, 27, 1 (2011), 52-60.

[Twining 2009] Twining, P. "Exploring the educational potential of virtual worlds-Some reflections from the. SPP”. British Journal of Educational Technology, 40, 3 (2009), 496-514.

[Tüzün 2007] Tüzün, H. "Blending video games with learning: Issues and challenges with classroom implementations in the Turkish context". British Journal of Educational Technology, 38, 3 (2007), 465-477.

[Toro-Troconis and Mellström 2010] Toro-Troconis, M. \& Mellström, U. "Game-based learning in Second Life $\circledast$. Do gender and age make a difference?” Journal of Gaming and Virtual Worlds, 1, 2 (2010), 53-76.

[Simsek 2016] Simsek, I. "The effect of 3D virtual learning environment on secondary school third grade students' attitudes toward mathematics". Turkish Online Journal of Educational Technology, 15, 3 (2016), $162-168$. 
[Wang \& Burton 2012] Wang, F. \& Burton, J. "Second Life in education: A review of publications from its launch to 2011". British Journal of Educational Technology, 44, 3 (2012), 357-371.

[Wang et al. 2018] Wang, X., Wanli, X. \& Laffey, J. "Autistic youth in 3D game-based collaborative virtual learning: Associating avatar interaction patterns with embodied social presence". British Journal of Educational Technology, 49, 4 (2018), 742-760.

[Yilmaz and Cagiltay 2016] Yilmaz, T. K. \& Cagiltay, K. "Designing and developing game-like learning experience in virtual worlds: Challenges and design decisions of novice instructional designers". Contemporary Educational Technology, 7,3 (2016), 206-222.

[Young et al. 2012] Young, W., Franklin, T., Cooper, T., Carroll, S., \& Liu, C. "Game-based learning aids in Second Life". Journal of Interactive Learning Research, 23, 1 (2012), 57-80.

[Zheng et al. 2009] Zheng, D., Young, M. \& Wagner, M. "Negotiation for action: English language learning in game-based virtual worlds". Modern Language Journal, 93, 4 (2009), 489-511. 